\title{
Association Between HDL Cholesterol and Incident Cognitive Impairment in Black and White Adults in The Reasons For Geographic and Racial Differences in Stroke Study
}

Robert Rosenson ( $\nabla$ robert.rosenson@mssm.edu )

Mount Sinai Hospital Mount Sinai Heart https://orcid.org/0000-0002-5599-0633

Mary Cushman

University of Vermont

Emily C. McKinley

The University of Alabama at Birmingham School of Medicine

Paul Muntner

University of Alabama at Birmingham

Zhixin Wang

University of Alabama at Birmingham

Tomas Vaisar

University of Washington

Jay Heinecke

University of Washington

Christy Tangney

Rush University

Suzanne Judd

University of Alabama

Lisandro D. Colantonio

University of Alabama at Birmingham

Research

Keywords: Cognitive impairment, dementia, high density lipoprotein cholesterol, triglycerides, stroke

Posted Date: October 22nd, 2021

DOl: https://doi.org/10.21203/rs.3.rs-961604/v1

License: (9) This work is licensed under a Creative Commons Attribution 4.0 International License.

Read Full License 
Page 2/15 


\section{Abstract \\ BACKGROUND:}

Low levels of high-density lipoprotein cholesterol (HDL-C) have been associated with incident cognitive impairment (ICl) in some, but not all studies.

\section{METHODS:}

We examine the association between HDL-C and ICl among 18,378 Reasons for Geographic and Racial Differences in Stroke (REGARDS) study participants without cognitive impairment or stroke at baseline in 2003 to 2007, and who did not have a stroke event during follow-up.

\section{RESULTS:}

Over a median follow-up of 9.6 years, 1,359 participants developed ICl. In fully adjusted models, the RR for ICl was $1.51(95 \% \mathrm{Cl} 1.06,2.15)$ in White women and 1.25 (95\% Cl 0.95, 1.65) in Black women. The association was not statistically significant afrter adjustment for triglycerides. There was no evidence of an association between HDL-C and ICl among White or Black men.

\section{CONCLUSIONS:}

Low HDL-C was associated with a higher risk of ICl in White and Black women in models not including triglycerides.

\section{Introduction:}

Low high-density lipoprotein cholesterol (HDL-C) has been associated with higher rates of incident cognitive impairment (ICI) in some, but not all, prior studies (1-5). The heterogeneity in the association between $\mathrm{HDL}-\mathrm{C}$ and $\mathrm{ICl}$ in prior studies may result from residual confounding by lifestyle, cardiovascular risk factors, systemic inflammation, and history of stroke (5-8). This study aimed to determine the association between HDL-C and ICl among race and sex subgroups in a population-based longitudinal cohort of White and Black middle-aged and older adults (9). The association between HDL-C and ICI was determined before and after adjustment for lifestyle behaviors, menopausal status and hormonal replacement therapies (HRT) in women, C-reactive protein, hypertension and triglycerides.

\section{Methods:}

Details on the design and methods of the REGARDS study have been published.(9) The Reasons for Geographic and Racial Differences in Stroke (REGARDS) study enrolled 30,239 US Black and White adults 
$\geq 45$ years of age between 2003 and 2007. Overall, $42 \%$ of enrolled participants were Black and $55 \%$ were women (9). The study was approved by the institutional review boards at all participating institutions, and all participants provided written informed consent.

Serum HDL-C was measured using blood collected at baseline by colorimetric reflectance spectrophotometry. For the current analysis, we included 26,977 study participants without $\mathrm{Cl}$, defined by a score $\geq 5$, at their first Six Item Screener (SIS) assessment. During follow-up, cognitive status was assessed using the SIS beginning in December 2003, and the three battery tests in the Consortium to Establish a Registry for Alzheimer's Disease (CERAD): Word List Learning, Word List Delayed Recall and Semantic Animal Fluency tests (10). CERAD and SIS tests were administered by telephone at 18-24 month and 12-month intervals, respectively. We excluded participants who did not complete $\geq 1$ assessment for each of the 3 CERAD battery tests during follow-up $(n=6,119)$, did not have valid data on HDL-C $(n=867)$, or who had a history of stroke at baseline or a stroke event during follow-up $(n=1,613)$. The final cohort included 18,378 participants. ICl was defined as a score $\geq 1.5$ standard deviations (SD) below the age, sex, and education-based stratum-specific means on 2 or 3 of the CERAD battery tests at any single determination during follow up.

\section{Statistical analysis}

We described participant characteristics and used Poisson regression models with robust variance estimators to calculate the risk ratio (RR) for ICl associated with HDL-C levels in a series of progressively adjusted models. We adjusted for income and education, smoking, second-hand smoke, body mass index, physical activity, alcohol consumption, use of HRT, and menopausal status among women. We further adjusted for systolic blood pressure and diagnosis of hypertension, and C-reactive protein (CRP). We adjusted for triglycerides due to inverse association with HDL-C. All analyses were conducted among White women, Black women, White men, and Black men, separately.

\section{Results:}

The characteristics of study participants and the distribution of HDL-C stratified by sex and race are presented in Table 1. Overall, $5.2 \%$ of White women, $11.6 \%$ of Black women, $5.8 \%$ of White men and $9.2 \%$ of Black men developed ICl over a median follow up of 9.6 years. After adjustment for age and region of residence (Model 1), HDL-C $<40 \mathrm{mg} / \mathrm{dL}$ versus $\geq 60 \mathrm{mg} / \mathrm{dL}$ was associated with an increased RR (95\% Confidence Interval $[\mathrm{Cl}])$ for $\mathrm{ICl}$ among White women $(1.60[1.16,2.21])$ and Black women $(1.35$ [1.03, 1.76], Table 2). HDL-C remained associated with ICl after additional adjustment for income and education in White women (RR 1.66 [95\% Cl 1.20, 2.31]) and Black women (RR 1.34 [95\% Cl 1.03, 1.75]). After further adjustment for body mass index, lifestyle factors (smoking, second-hand smoke, body mass index, physical activity, alcohol consumption), menopausal status, and hormone replacement therapy, the RR for ICl was $1.51(95 \% \mathrm{Cl} 1.06,2.15)$ in White women and 1.25 (95\% Cl 0.95, 1.65) in Black women. The association between a low HDL-C and ICI was attenuated after additional adjustment for triglycerides 
(White women: RR 1.31 [95\% Cl 0.89, 1.94], Black women: RR 1.12 [95\% Cl 0.84, 1.51) ]). There was no evidence of associations between HDL-C and ICl in Black or White men. 
Table 1

Characteristics of REGARDS study participants stratified by sex and race.

Women

White

$(n=6,222)$
Black

$(n=4,259)$
Men

White

$(n=5,588)$

Black

$(n=2,309)$

\section{Characteristics}

\begin{tabular}{|c|c|c|c|c|}
\hline Age, years, mean (SD) & $63.6(9.1)$ & $62.7(8.6)$ & $64.5(8.7)$ & $63.0(8.6)$ \\
\hline \multicolumn{5}{|l|}{ Age, years } \\
\hline $45-54$ & $941(15.1 \%)$ & $675(15.8 \%)$ & $596(10.7 \%)$ & $349(15.1 \%)$ \\
\hline $55-64$ & $\begin{array}{l}2530 \\
(40.7 \%)\end{array}$ & $\begin{array}{l}1882 \\
(44.2 \%)\end{array}$ & $\begin{array}{l}2303 \\
(41.2 \%)\end{array}$ & $\begin{array}{l}1005 \\
(43.5 \%)\end{array}$ \\
\hline $65-74$ & $\begin{array}{l}1889 \\
(30.4 \%)\end{array}$ & $\begin{array}{l}1274 \\
(29.9 \%)\end{array}$ & $\begin{array}{l}1914 \\
(34.3 \%)\end{array}$ & $715(31.0 \%)$ \\
\hline$\geq 75$ & $862(13.9 \%)$ & $428(10.0 \%)$ & $775(13.9 \%)$ & $240(10.4 \%)$ \\
\hline \multicolumn{5}{|l|}{ Education level, n (\%) } \\
\hline No high school graduate & $381(6.1 \%)$ & $641(15.1 \%)$ & $252(4.5 \%)$ & $276(12.0 \%)$ \\
\hline High school graduate & $\begin{array}{l}1673 \\
(26.9 \%)\end{array}$ & $\begin{array}{l}1146 \\
(26.9 \%)\end{array}$ & $\begin{array}{l}1074 \\
(19.2 \%)\end{array}$ & $611(26.5 \%)$ \\
\hline Some college education & $\begin{array}{l}1767 \\
(28.4 \%)\end{array}$ & $\begin{array}{l}1200 \\
(28.2 \%)\end{array}$ & $\begin{array}{l}1330 \\
(23.8 \%)\end{array}$ & $668(28.9 \%)$ \\
\hline College graduate or higher & $\begin{array}{l}2400 \\
(38.6 \%)\end{array}$ & $\begin{array}{l}1269 \\
(29.8 \%)\end{array}$ & $\begin{array}{l}2931 \\
(52.5 \%)\end{array}$ & $754(32.7 \%)$ \\
\hline \multicolumn{5}{|c|}{ Annual household Income, n (\%) } \\
\hline$<\$ 20,000$ & $806(13.0 \%)$ & $\begin{array}{l}1094 \\
(25.7 \%)\end{array}$ & $295(5.3 \%)$ & $297(12.9 \%)$ \\
\hline$\$ 20,000-34,000$ & $\begin{array}{l}1475 \\
(23.7 \%)\end{array}$ & $\begin{array}{l}1102 \\
(25.9 \%)\end{array}$ & $989(17.7 \%)$ & $579(25.1 \%)$ \\
\hline$\$ 35,000-74,000$ & $\begin{array}{l}1887 \\
(30.3 \%)\end{array}$ & $\begin{array}{l}1200 \\
(28.2 \%)\end{array}$ & $\begin{array}{l}2188 \\
(39.2 \%)\end{array}$ & $833(36.1 \%)$ \\
\hline$\geq \$ 75,000$ & $\begin{array}{l}1126 \\
(18.1 \%)\end{array}$ & $367(8.6 \%)$ & $\begin{array}{l}1671 \\
(29.9 \%)\end{array}$ & $412(17.8 \%)$ \\
\hline Declined to report & $928(14.9 \%)$ & $496(11.6 \%)$ & $445(8.0 \%)$ & $188(8.1 \%)$ \\
\hline
\end{tabular}




\begin{tabular}{|c|c|c|c|c|}
\hline & Women & & Men & \\
\hline Stroke belt & $\begin{array}{l}2232 \\
(35.9 \%)\end{array}$ & $\begin{array}{l}1390 \\
(32.6 \%)\end{array}$ & $\begin{array}{l}1911 \\
(34.2 \%)\end{array}$ & $765(33.1 \%)$ \\
\hline Stroke buckle & $\begin{array}{l}1593 \\
(25.6 \%)\end{array}$ & $833(19.6 \%)$ & $\begin{array}{l}1143 \\
(20.5 \%)\end{array}$ & $389(16.8 \%)$ \\
\hline Non-belt region & $\begin{array}{l}2397 \\
(38.5 \%)\end{array}$ & $\begin{array}{l}2036 \\
(47.8 \%)\end{array}$ & $\begin{array}{l}2534 \\
(45.3 \%)\end{array}$ & $\begin{array}{l}1155 \\
(50.0 \%)\end{array}$ \\
\hline \multicolumn{5}{|l|}{ Smoke Status, n (\%) } \\
\hline Current smoker & $750(12.1 \%)$ & $599(14.1 \%)$ & $558(10.0 \%)$ & $378(16.5 \%)$ \\
\hline $\begin{array}{l}\text { No current smoker, exposed to } \\
\text { secondhand smoke }\end{array}$ & $\begin{array}{l}3468 \\
(55.9 \%)\end{array}$ & $\begin{array}{l}2303 \\
(54.3 \%)\end{array}$ & $\begin{array}{l}2198 \\
(39.5 \%)\end{array}$ & $825(35.9 \%)$ \\
\hline $\begin{array}{l}\text { No current smoker, not exposed to } \\
\text { secondhand smoke }\end{array}$ & $\begin{array}{l}1981 \\
(32.0 \%)\end{array}$ & $\begin{array}{l}1340 \\
(31.6 \%)\end{array}$ & $\begin{array}{l}2815 \\
(50.5 \%)\end{array}$ & $\begin{array}{l}1093 \\
(47.6 \%)\end{array}$ \\
\hline Diabetes, n (\%) & $680(11.0 \%)$ & $\begin{array}{l}1059 \\
(25.0 \%)\end{array}$ & $826(14.9 \%)$ & $615(26.8 \%)$ \\
\hline \multicolumn{5}{|l|}{ Body mass index, $\mathrm{n}(\%)$} \\
\hline$<18.5-24.9 \mathrm{Kg} / \mathrm{m}^{2}$ & $\begin{array}{l}2103 \\
(33.9 \%)\end{array}$ & $572(13.5 \%)$ & $\begin{array}{l}1294 \\
(23.3 \%)\end{array}$ & $467(20.4 \%)$ \\
\hline $25-29.9 \mathrm{Kg} / \mathrm{m}^{2}$ & $\begin{array}{l}2074 \\
(33.5 \%)\end{array}$ & $\begin{array}{l}1259 \\
(29.8 \%)\end{array}$ & $\begin{array}{l}2580 \\
(46.5 \%)\end{array}$ & 925 (40.4\%) \\
\hline$\geq 30 \mathrm{Kg} / \mathrm{m}^{2}$ & $\begin{array}{l}2018 \\
(32.6 \%)\end{array}$ & $\begin{array}{l}2397 \\
(56.7 \%)\end{array}$ & $\begin{array}{l}1680 \\
(30.2 \%)\end{array}$ & $900(39.3 \%)$ \\
\hline $\begin{array}{l}\text { Systolic blood pressure, } \mathrm{mmHg} \text {, mean } \\
\text { (SD) }\end{array}$ & $122.5(15.4)$ & $\begin{array}{l}128.7 \\
(16.5)\end{array}$ & $126.2(14.8)$ & $\begin{array}{l}130.9 \\
(16.0)\end{array}$ \\
\hline $\begin{array}{l}\text { Diastolic blood pressure, } \mathrm{mmHg} \text {, mean } \\
\text { (SD) }\end{array}$ & $74.2(8.9)$ & $77.9(9.7)$ & $76.3(9.1)$ & $79.4(9.7)$ \\
\hline \multicolumn{5}{|l|}{ Physical Activity, n (\%) } \\
\hline$\geq 4$ bouts/week of intense activity & $\begin{array}{l}1623 \\
(26.4 \%)\end{array}$ & $982(23.3 \%)$ & $\begin{array}{l}2129 \\
(38.5 \%)\end{array}$ & $720(31.6 \%)$ \\
\hline $1-3$ bouts/week of intense activity & $\begin{array}{l}2371 \\
(38.6 \%)\end{array}$ & $\begin{array}{l}1616 \\
(38.4 \%)\end{array}$ & $\begin{array}{l}2121 \\
(38.4 \%)\end{array}$ & $948(41.6 \%)$ \\
\hline 0 bouts/week of intense activity & $\begin{array}{l}2148 \\
(35.0 \%)\end{array}$ & $\begin{array}{l}1615 \\
(38.3 \%)\end{array}$ & $\begin{array}{l}1274 \\
(23.1 \%)\end{array}$ & $613(26.9 \%)$ \\
\hline \multicolumn{5}{|l|}{ Alcohol consumption, $\mathrm{n}(\%)^{\mathrm{b}}$} \\
\hline None & $\begin{array}{l}3766 \\
(61.3 \%)\end{array}$ & $\begin{array}{l}3174 \\
(76.1 \%)\end{array}$ & $\begin{array}{l}2491 \\
(45.3 \%)\end{array}$ & $\begin{array}{l}1276 \\
(56.6 \%)\end{array}$ \\
\hline
\end{tabular}




\begin{tabular}{|c|c|c|c|c|}
\hline & Women & & Men & \\
\hline Moderate & $\begin{array}{l}2069 \\
(33.7 \%)\end{array}$ & $927(22.2 \%)$ & $\begin{array}{l}2704 \\
(49.2 \%)\end{array}$ & $899(39.9 \%)$ \\
\hline Heavy & $311(5.1 \%)$ & $69(1.7 \%)$ & $306(5.6 \%)$ & $78(3.5 \%)$ \\
\hline $\begin{array}{l}\text { Triglycerides, } \mathrm{mg} / \mathrm{dL} \text {, median }(25 \% \text {, } \\
75 \% \text { percentiles) }\end{array}$ & $\begin{array}{l}120.0 \\
(87.0,169.0)\end{array}$ & $\begin{array}{l}94.0 \\
(73.0,126.0)\end{array}$ & $\begin{array}{l}122.0 \\
(88.0,176.0)\end{array}$ & $\begin{array}{l}97.0 \\
(73.0,137.0)\end{array}$ \\
\hline C-Reactive Protein $\geq 2$ mg/L, n (\%) & $\begin{array}{l}3175 \\
(52.4 \%)\end{array}$ & $\begin{array}{l}2744 \\
(66.3 \%)\end{array}$ & $\begin{array}{l}2077 \\
(37.9 \%)\end{array}$ & $\begin{array}{l}1119 \\
(49.3 \%)\end{array}$ \\
\hline Menopause, n (\%) & $\begin{array}{l}5505 \\
(90.0 \%)\end{array}$ & $\begin{array}{l}3695 \\
(89.4 \%)\end{array}$ & - & - \\
\hline Antihypertensive therapy, n (\%) & $\begin{array}{l}2989 \\
(48.0 \%)\end{array}$ & $\begin{array}{l}2892 \\
(67.9 \%)\end{array}$ & $\begin{array}{l}2808 \\
(50.3 \%)\end{array}$ & $\begin{array}{l}1453 \\
(62.9 \%)\end{array}$ \\
\hline Statin therapy, n (\%) & $\begin{array}{l}1686 \\
(27.1 \%)\end{array}$ & $\begin{array}{l}1144 \\
(26.9 \%)\end{array}$ & $\begin{array}{l}2053 \\
(36.7 \%)\end{array}$ & $681(29.5 \%)$ \\
\hline Hormone replacement therapy, $\mathrm{n}(\%)$ & $\begin{array}{l}4183 \\
(67.2 \%)\end{array}$ & $\begin{array}{l}2054 \\
(48.2 \%)\end{array}$ & $61(1.1 \%)$ & $13(0.6 \%)$ \\
\hline $\begin{array}{l}\text { Baseline Six-Item Screener score, } \\
\text { mean (SD) }\end{array}$ & $5.9(0.3)$ & $5.7(0.4)$ & $5.8(0.4)$ & $5.7(0.5)$ \\
\hline HDL-C, mg/dL, mean (SD) & $58.0(16.4)$ & $57.3(15.8)$ & $44.3(12.9)$ & $48.0(14.3)$ \\
\hline \multicolumn{5}{|l|}{$\begin{array}{l}\text { HDL-C categories among women, } n \\
(\%)\end{array}$} \\
\hline$<40 \mathrm{mg} / \mathrm{dL}$ & $693(11.1 \%)$ & 419 (9.8\%) & - & - \\
\hline $40-49 \mathrm{mg} / \mathrm{dL}$ & $\begin{array}{l}1374 \\
(22.1 \%)\end{array}$ & $\begin{array}{l}1044 \\
(24.5 \%)\end{array}$ & - & - \\
\hline $50-59 \mathrm{mg} / \mathrm{dL}$ & $\begin{array}{l}1586 \\
(25.5 \%)\end{array}$ & $\begin{array}{l}1164 \\
(27.3 \%)\end{array}$ & - & - \\
\hline$\geq 60 \mathrm{mg} / \mathrm{dL}$ & $\begin{array}{l}2569 \\
(41.3 \%)\end{array}$ & $\begin{array}{l}1632 \\
(38.3 \%)\end{array}$ & - & - \\
\hline \multicolumn{5}{|l|}{ HDL-C categories among men, $\mathrm{n}(\%)$} \\
\hline$<30 \mathrm{mg} / \mathrm{dL}$ & - & - & $463(8.3 \%)$ & $102(4.4 \%)$ \\
\hline $30-39 \mathrm{mg} / \mathrm{dL}$ & - & - & $\begin{array}{l}1752 \\
(31.4 \%)\end{array}$ & $573(24.8 \%)$ \\
\hline $40-49 \mathrm{mg} / \mathrm{dL}$ & - & - & $\begin{array}{l}1798 \\
(32.2 \%)\end{array}$ & $762(33.0 \%)$ \\
\hline$\geq 50 \mathrm{mg} / \mathrm{dL}$ & - & - & $\begin{array}{l}1575 \\
(28.2 \%)\end{array}$ & $872(37.8 \%)$ \\
\hline
\end{tabular}


HDL-C: high-density lipoprotein cholesterol.

Numbers reported in table are $\mathrm{n}(\%)$ or mean (SD).

a Stroke buckle includes coastal North Carolina, South Carolina and Georgia. Stroke belt includes the remaining parts of North Carolina, South Carolina and Georgia, and Tennessee, Mississippi, Alabama, Louisiana and Arkansas. Other US regions includes the remaining 40 contiguous US states and the District of Columbia.

${ }^{b}$ Based on the number of drinks that participants self-reported having per week during their baseline computer-assisted telephone interview and categorized as follows:

- No alcohol consumption: 0 drinks per week.

- Moderate alcohol consumption: $>0$ to 7 drinks per week for women and $>0$ to 14 drinks per week for men.

- Heavy alcohol consumption: $>7$ drinks per week for women and >14 drinks per week for men. 
Table 2

Relative risk for $\mathrm{ICl}$ associated with $\mathrm{HDL}-\mathrm{C}$ levels, stratified by sex and race

\begin{tabular}{|c|c|c|c|c|c|c|c|}
\hline \multirow{2}{*}{$\begin{array}{l}\text { HDL-C } \\
\text { Level }\end{array}$} & \multirow{2}{*}{$\begin{array}{l}\mathrm{N}(\%) \text { with ICl / Total } \\
\text { number of participants }\end{array}$} & \multicolumn{6}{|c|}{ Risk ratio ( $95 \%$ confidence interval) } \\
\hline & & $\begin{array}{l}\text { Model } \\
1\end{array}$ & $\begin{array}{l}\text { Model } \\
2\end{array}$ & $\begin{array}{l}\text { Model } \\
3\end{array}$ & $\begin{array}{l}\text { Model } \\
4 \mathrm{a}\end{array}$ & $\begin{array}{l}\text { Model } \\
4 \mathrm{~b}\end{array}$ & $\begin{array}{l}\text { Model } \\
4 \mathrm{c}\end{array}$ \\
\hline & \multicolumn{7}{|l|}{ White Females } \\
\hline $\begin{array}{l}<40 \\
\mathrm{mg} / \mathrm{dL}\end{array}$ & $49(7.1 \%) / 693$ & $\begin{array}{l}1.60 \\
(1.16 \\
2.21)\end{array}$ & $\begin{array}{l}1.66 \\
(1.20 \\
2.31)\end{array}$ & $\begin{array}{l}1.51 \\
(1.06 \\
2.15)\end{array}$ & $\begin{array}{l}1.65 \\
(1.16 \\
2.35)\end{array}$ & $\begin{array}{l}1.48 \\
(1.04 \\
2.10)\end{array}$ & $\begin{array}{l}1.31 \\
(0.89 \\
1.94)\end{array}$ \\
\hline $\begin{array}{l}40-49 \\
\mathrm{mg} / \mathrm{dL}\end{array}$ & $76(5.5 \%) / 1,374$ & $\begin{array}{l}1.24 \\
(0.94 \\
1.65)\end{array}$ & $\begin{array}{l}1.27 \\
(0.96 \\
1.69)\end{array}$ & $\begin{array}{l}1.20 \\
(0.89 \\
1.62)\end{array}$ & $\begin{array}{l}1.26 \\
(0.93 \\
1.70)\end{array}$ & $\begin{array}{l}1.19 \\
(0.88 \\
1.60)\end{array}$ & $\begin{array}{l}1.10 \\
(0.80 \\
1.51)\end{array}$ \\
\hline $\begin{array}{l}50-59 \\
\mathrm{mg} / \mathrm{dL}\end{array}$ & $87(5.5 \%) / 1,586$ & $\begin{array}{l}1.23 \\
(0.94 \\
1.61)\end{array}$ & $\begin{array}{l}1.27 \\
(0.97 \\
1.67)\end{array}$ & $\begin{array}{l}1.23 \\
(0.92 \\
1.63)\end{array}$ & $\begin{array}{l}1.25 \\
(0.94 \\
1.67)\end{array}$ & $\begin{array}{l}1.22 \\
(0.92 \\
1.62)\end{array}$ & $\begin{array}{l}1.17 \\
(0.88 \\
1.56)\end{array}$ \\
\hline \multirow{2}{*}{$\begin{array}{l}\geq 60 \\
\mathrm{mg} / \mathrm{dL}\end{array}$} & $114(4.4 \%) / 2,569$ & 1 (ref) & 1 (ref) & 1 (ref) & 1 (ref) & 1 (ref) & 1 (ref) \\
\hline & \multicolumn{7}{|l|}{ Black Females } \\
\hline $\begin{array}{l}<40 \\
\mathrm{mg} / \mathrm{dL}\end{array}$ & $61(14.6 \%) / 419$ & $\begin{array}{l}1.35 \\
(1.03, \\
1.76)\end{array}$ & $\begin{array}{l}1.34 \\
(1.03 \\
1.75)\end{array}$ & $\begin{array}{l}1.25 \\
(0.95 \\
1.65)\end{array}$ & $\begin{array}{l}1.24 \\
(0.93 \\
1.64)\end{array}$ & $\begin{array}{l}1.22 \\
(0.92 \\
1.62)\end{array}$ & $\begin{array}{l}1.12 \\
(0.84 \\
1.51)\end{array}$ \\
\hline $\begin{array}{l}40-49 \\
\mathrm{mg} / \mathrm{dL}\end{array}$ & $120(11.5 \%) / 1,044$ & $\begin{array}{l}1.05 \\
(0.84 \\
1.30)\end{array}$ & $\begin{array}{l}1.05 \\
(0.85 \\
1.30)\end{array}$ & $\begin{array}{l}0.97 \\
(0.77 \\
1.22)\end{array}$ & $\begin{array}{l}0.92 \\
(0.73 \\
1.17)\end{array}$ & $\begin{array}{l}0.95 \\
(0.76 \\
1.20)\end{array}$ & $\begin{array}{l}0.90 \\
(0.71 \\
1.15)\end{array}$ \\
\hline $\begin{array}{l}50-59 \\
\mathrm{mg} / \mathrm{dL}\end{array}$ & $132(11.3 \%) / 1,164$ & $\begin{array}{l}1.03 \\
(0.83 \\
1.27)\end{array}$ & $\begin{array}{l}1.04 \\
(0.84 \\
1.29)\end{array}$ & $\begin{array}{l}1.00 \\
(0.80 \\
1.25)\end{array}$ & $\begin{array}{l}1.00 \\
(0.80 \\
1.25)\end{array}$ & $\begin{array}{l}1.00 \\
(0.80 \\
1.25)\end{array}$ & $\begin{array}{l}0.96 \\
(0.77 \\
1.20)\end{array}$ \\
\hline \multirow{2}{*}{$\begin{array}{l}\geq 60 \\
\mathrm{mg} / \mathrm{dL}\end{array}$} & $182(11.2 \%) / 1,632$ & 1 (ref) & 1 (ref) & 1 (ref) & 1 (ref) & 1 (ref) & 1 (ref) \\
\hline & \multicolumn{7}{|l|}{ White Males } \\
\hline $\begin{array}{l}<30 \\
\mathrm{mg} / \mathrm{dL}\end{array}$ & $22(4.8 \%) / 463$ & $\begin{array}{l}0.96 \\
(0.61, \\
1.52)\end{array}$ & $\begin{array}{l}0.99 \\
(0.63 \\
1.57)\end{array}$ & $\begin{array}{l}0.89 \\
(0.55 \\
1.43)\end{array}$ & $\begin{array}{l}0.92 \\
(0.57 \\
1.49)^{\prime}\end{array}$ & $\begin{array}{l}0.87 \\
(0.54, \\
1.40)\end{array}$ & $\begin{array}{l}0.82 \\
(0.49 \\
1.35)\end{array}$ \\
\hline $\begin{array}{l}30-39 \\
\mathrm{mg} / \mathrm{dL}\end{array}$ & $119(6.8 \%) / 1,752$ & $\begin{array}{l}1.34 \\
(1.02 \\
1.76)\end{array}$ & $\begin{array}{l}1.38 \\
(1.05 \\
1.83)\end{array}$ & $\begin{array}{l}1.25 \\
(0.93 \\
1.70)\end{array}$ & $\begin{array}{l}1.29 \\
(0.95 \\
1.75)\end{array}$ & $\begin{array}{l}1.23 \\
(0.91 \\
1.67)\end{array}$ & $\begin{array}{l}1.19 \\
(0.85, \\
1.66)\end{array}$ \\
\hline $\begin{array}{l}40-49 \\
\mathrm{mg} / \mathrm{dL}\end{array}$ & $104(5.8 \%) / 1,798$ & $\begin{array}{l}1.14 \\
(0.86 \\
1.52)\end{array}$ & $\begin{array}{l}1.16 \\
(0.88 \\
1.54)\end{array}$ & $\begin{array}{l}1.05 \\
(0.77 \\
1.42)\end{array}$ & $\begin{array}{l}1.07 \\
(0.79 \\
1.45)\end{array}$ & $\begin{array}{l}1.04 \\
(0.77 \\
1.41)\end{array}$ & $\begin{array}{l}1.02 \\
(0.74 \\
1.39)\end{array}$ \\
\hline $\begin{array}{l}\geq 50 \\
\mathrm{mg} / \mathrm{dL}\end{array}$ & $80(5.1 \%) / 1,575$ & 1 (ref) & 1 (ref) & 1 (ref) & 1 (ref) & 1 (ref) & 1 (ref) \\
\hline
\end{tabular}




\begin{tabular}{|c|c|c|c|c|c|c|c|}
\hline \multirow{2}{*}{$\begin{array}{l}\text { HDL-C } \\
\text { Level }\end{array}$} & \multirow{2}{*}{$\begin{array}{l}\mathrm{N}(\%) \text { with } \mathrm{ICl} / \text { Total } \\
\text { number of participants }\end{array}$} & \multicolumn{6}{|c|}{ Risk ratio ( $95 \%$ confidence interval) } \\
\hline & & $\begin{array}{l}\text { Model } \\
1\end{array}$ & $\begin{array}{l}\text { Model } \\
2\end{array}$ & $\begin{array}{l}\text { Model } \\
3\end{array}$ & $\begin{array}{l}\text { Model } \\
4 a\end{array}$ & $\begin{array}{l}\text { Model } \\
4 \mathrm{~b}\end{array}$ & $\begin{array}{l}\text { Model } \\
4 c\end{array}$ \\
\hline & \multicolumn{7}{|l|}{ Black Males } \\
\hline $\begin{array}{l}<30 \\
\mathrm{mg} / \mathrm{dL}\end{array}$ & $8(7.8 \%) / 102$ & $\begin{array}{l}0.80 \\
(0.40 \\
1.61)\end{array}$ & $\begin{array}{l}0.81 \\
(0.40 \\
1.64)\end{array}$ & $\begin{array}{l}0.75 \\
(0.37 \\
1.53)\end{array}$ & $\begin{array}{l}0.78 \\
(0.39 \\
1.59)\end{array}$ & $\begin{array}{l}0.74 \\
(0.36, \\
1.51)\end{array}$ & $\begin{array}{l}0.73 \\
(0.35 \\
1.52)\end{array}$ \\
\hline $\begin{array}{l}30-39 \\
\mathrm{mg} / \mathrm{dL}\end{array}$ & $53(9.2 \%) / 573$ & $\begin{array}{l}0.95 \\
(0.68 \\
1.31)\end{array}$ & $\begin{array}{l}0.93 \\
(0.68 \\
1.29)\end{array}$ & $\begin{array}{l}0.88 \\
(0.63 \\
1.24)\end{array}$ & $\begin{array}{l}0.86 \\
(0.61 \\
1.22)\end{array}$ & $\begin{array}{l}0.87 \\
(0.62 \\
1.23)\end{array}$ & $\begin{array}{l}0.87 \\
(0.60 \\
1.26)\end{array}$ \\
\hline $\begin{array}{l}40-49 \\
\mathrm{mg} / \mathrm{dL}\end{array}$ & $67(8.8 \%) / 762$ & $\begin{array}{l}0.90 \\
(0.66 \\
1.22)\end{array}$ & $\begin{array}{l}0.93 \\
(0.68 \\
1.25)\end{array}$ & $\begin{array}{l}0.83 \\
(0.60 \\
1.15)\end{array}$ & $\begin{array}{l}0.82 \\
(0.59 \\
1.14)\end{array}$ & $\begin{array}{l}0.82 \\
(0.59 \\
1.14)\end{array}$ & $\begin{array}{l}0.82 \\
(0.59 \\
1.15)\end{array}$ \\
\hline $\begin{array}{l}\geq 50 \\
\mathrm{mg} / \mathrm{dL}\end{array}$ & $85(9.7 \%) / 872$ & 1 (ref) & 1 (ref) & 1 (ref) & 1 (ref) & 1 (ref) & 1 (ref) \\
\hline \multicolumn{8}{|c|}{ Data are presented as relative risks and $95 \%$ confidence intervals. } \\
\hline \multicolumn{8}{|c|}{ ICl: Incident cognitive impairment; HDL-C: high-density lipoprotein cholesterol } \\
\hline \multicolumn{8}{|c|}{$\begin{array}{l}\text { ICl was defined by a score } 1.5 \text { standard deviations below the age, sex, and education adjusted mean } \\
\text { on at least two of the three tests in the Three Test Battery: Word List Learning, Word List Delayed } \\
\text { Recall, and Animal Naming Tests at any time during follow-up. }\end{array}$} \\
\hline \multicolumn{8}{|c|}{ Model 1 adjusts for age and geographic region of residence. } \\
\hline \multicolumn{8}{|c|}{ Model 2 adjusts for the components of Model 1 plus income and education. } \\
\hline \multicolumn{8}{|c|}{$\begin{array}{l}\text { Model } 3 \text { adjusts for the components of Model } 2 \text { plus smoking, second-hand smoke, body mass index, } \\
\text { physical activity, alcohol consumption, use of hormone replacement therapy, and menopausal status } \\
\text { among women. }\end{array}$} \\
\hline \multicolumn{8}{|c|}{ Model $4 a$ adjusts for variables in Model 3 plus C-reactive protein. } \\
\hline \multicolumn{8}{|c|}{$\begin{array}{l}\text { Model } 4 \mathrm{~b} \text { adjusts for variables in Model } 3 \text { plus systolic blood pressure and hypertension medication } \\
\text { use. }\end{array}$} \\
\hline \multicolumn{8}{|c|}{ Model $4 \mathrm{c}$ adjusts for variables in Model 3 plus the log of triglycerides. } \\
\hline
\end{tabular}

\section{Discussion:}

In this large prospective, population-based study of White and Black women and men, low HDL-C ( $<40$ $\mathrm{mg} / \mathrm{dL}$ ) was associated with an increased risk for $\mathrm{ICl}$ in White and Black women, but not in men. The higher risk of ICl was present before and after adjustment for factors known to contribute to HDL-C. However, adjustment for triglycerides attenuated these associations in both White women and Black women. Examination of race-sex differences is important because of well-known differences in levels of HDL-C between women and men and triglyceride levels between White and Black people (11). 
Multiple prospective population-based studies, $(1,3,4)$ and a pooled analysis of 11 cohort studies including 5,658 participants, reported that lower levels of HDL-C were associated with cognitive decline (10). These studies included participants with a history of stroke. In contrast, there was no association between HDL-C and cognitive function in an analysis of 5,033 stroke-free women and men who participated in the Tromso study (5). The results from the current study are consistent with the findings from the Tromso study.

Few studies have analyzed sex-specific differences in HDL-C and ICI. The China Health and Retirement Longitudinal Study (CHARLS) investigated ICl in 6,792 Chinese stroke-free adults aged 45 years and older, and paradoxically reported a higher HDL-C level was associated with a higher risk of cognitive decline in women, but a lower risk of cognitive decline in men (12). Among women $<60$ years of age, the adjusted odds ratio for cognitive decline associated with HDL-C levels of $40-59 \mathrm{mg} / \mathrm{dL}$ and $\geq 60 \mathrm{mg} / \mathrm{dL}$ versus $<40$ $\mathrm{mg} / \mathrm{dL}$ was $1.37(95 \% \mathrm{Cl} 1.04,1.80)$ and $1.29(95 \% \mathrm{Cl} 0.94,1.77)$, respectively. Episodic memory loss was also associated with a higher HDL-C in women (adjusted odds ratio associated with HDL-C 40-59 mg/dL and $\geq 60 \mathrm{mg} / \mathrm{dL}$ versus $<40 \mathrm{mg} / \mathrm{dL} 1.55$ [95\% Cl 1.18, 2.03] and 1.55 [95\% Cl 1.13, 2. 13], respectively). Fasting triglyceride levels were not associated with cognitive decline in participants in CHARLS. In the REGARDS study, an increased risk of ICI in Black and White women with low HDL-C concentrations was present after multivariate adjustment with the exception of triglycerides. Elevated triglycerides were associated with an increased risk of non-Alzheimer's dementia and stroke in a prospective study of 125,727 individuals participating in the Copenhagen General Population Study and Copenhagen City Heart Study (13). Triglyceride-rich lipoproteins increase atherosclerosis contributing to increased risk of stroke and non-Alzheimer's dementia in Blacks and Whites, but not apparently in Chinese.

HDL-C is a common metric for HDL; however, the cholesterol content of HDL is an inadequate metric for the multifarious functions of HDL (14). Emerging evidence from genetic, clinical and experimental studies supports the involvement of surface protein components of HDL in cognitive function (15). In the current study, we were unable to determine the association of HDL-C with the plethora of anti-oxidant and antiinflammatory properties of specific HDL subspecies that may have important physiological involvement in mitigating the risk of cognitive decline (14). However, the association between a low level of HDL-C in White women remained present after adjustment for the systemic inflammatory marker CRP.

\section{LIMITATIONS:}

$\mathrm{Cl}$ and dementia have various etiologies. Specific causes of $\mathrm{Cl}$ or dementia were unavailable in this cohort analysis. The REGARDS study is limited to Black and White Amercians; therefore, the results may not be generalizable to other race/ethnic groups including Asians or Hispanics. We used data on HDL-C as a surrogate marker for HDL, while recognizing the importance of HDL proteins in cholesterol efflux and mitigating inflammation. An ongoing analysis of REGARDS (NIH R01AG061186) is evaluating the HDL proteome in $\mathrm{ICl}$ and Alzheimer's dementia. Other limitations include the lack of data on triglyceride-rich lipoproteins, which also contribute to vascular inflammation. Markers of inflammation were limited to CRP.

Page $12 / 15$ 


\section{Conclusion:}

In conclusion, low HDL-C was associated with ICl in White and Black women. Adjustment for lifestyle factors partially attenuated the association between low HDL-C and ICI. Triglyceride-rich lipoproteins share many of the same determinants as $\mathrm{HDL}-\mathrm{C}$, but have distinct properties that may contribute to ICI.

\section{Declarations}

\section{Ethics approval and consent to participate}

Study methods were approved by the institutional review boards at participating institutions and all participants provided written informed consent for the storage of their clinical data for research purposes as part of the Reasons for Geographic and Racial Differences in Stroke (REGARDS) project.

\section{Consent for publication:}

Not applicable

\section{Availability of data and materials}

Qualified researchers trained in human subject confidentiality protocols may request access to the data that support the findings of this study by contacting the REGARDS Study at REGARDS@uab.edu

\section{Competing interests:}

The following authors received support for this work:

RSR, MC, PM, TV, SJ, and LDC.

RSR reports research funding to his institution from NIH, Amgen, Arrowhead, Novartis and Regeneron, consulting fees from Amgen, Amryt, C5, CVS Caremark, Novartis and Regneron, non-promotional speaking fees from Amgen, Kowa and Regeneron, royalties from Wolters Kluwer (UpToDate), and stock holdings in MediMergent, LLC.

MC reports NIH grants to institution, Data Safety Monitoring Board or Advisory Board for a CIHR funded clinical trial on statin use in venous thrombosis, unpaid leadership role on Hemostasis and Thrombosis Research Society board, and Journal Editor in Chief stipend from the International Society on Thrombosis and Haemotsasis.

ECM reports Amgen funding to institution.

PM reports NIH funding to institution, Amgen funding to institution, and Amgen consulting fees.

ZW reports no disclosures. 
TV reports NIH funding to institution, unrelated Astrazeneca consulting fees, Education event payment from ICOLA 2021 to self, pending patent for LCMSMS method for quantification of Lp(a).

$\mathrm{JH}$ reports $\mathrm{NIH}$ MPI and NIH PPG grant funding to institution, and expert testimony for a patent case.

CT reports grants to institution, royalties from Wolters Kluwer (UpToDate) to self, educational event payment for Lecture on Nutrition and Parkinson's Disease to self, and a leadership role in the Arlington Heights Memorial Library Board of Trustees.

SJ reports NIH funding to institution.

LDC reports Amgen funding to institution.

\section{Funding Sources:}

This study was supported by NIH R01AG061186. REGARDS is supported by cooperative agreement U01 NS041588 co-funded by the National Institute of Neurological Disorders and Stroke (NINDS) and the National Institute on Aging (NIA), National Institutes of Health, Department of Health and Human Service. The content is solely the responsibility of the authors and does not necessarily represent the official views of the NINDS or the NIA. Representatives of the NINDS were involved in the review of the manuscript but were not directly involved in the collection, management, analysis or interpretation of the data.

\section{Authors' contributions}

The individual contributions of authors to the manuscript should be specified in this section. Guidance and criteria for authorship can be found in our editorial policies.

RSR, MC, PM, LDC, JH obtained funding for this research. RSR, MC, EM, PM, TV, CT, SJ, LDC analyzed and interpreted the data and wrote the manuscript, ZW conducted the statistical analyses. All authors read and approved the final manuscript.

\section{Acknowledgements}

We acknowledge Ying Wen for contributing to part of the programming and data analyses, and Priscilla Meija and Shaunte Baboumian for assistance with manuscript preparation. The authors thank the other investigators, the staff, and the participants of the REGARDS study for their valuable contributions. A full list of participating REGARDS investigators and institutions can be found at:

https://www.uab.edu/soph/regardsstudy/

\section{References}

1. Solomon A, Kareholt I, Ngandu T, Winblad B, Nissinen A, Tuomilehto J, et al. Serum cholesterol changes after midlife and late-life cognition: twenty-one-year follow-up study. Neurology. 2007;68(10):751-6. 
2. Kaffashian S, Dugravot A, Nabi H, Batty GD, Brunner E, Kivimaki M, et al. Predictive utility of the Framingham general cardiovascular disease risk profile for cognitive function: evidence from the Whitehall II study. Eur Heart J. 2011;32(18):2326-32.

3. Crichton GE, Elias MF, Davey A, Sullivan KJ, Robbins MA. Higher HDL cholesterol is associated with better cognitive function: the Maine-Syracuse study. J Int Neuropsychol Soc. 2014;20(10):961-70.

4. Corley J, Starr JM, Deary IJ. Serum cholesterol and cognitive functions: the Lothian Birth Cohort 1936. Int Psychogeriatr. 2015;27(3):439-53.

5. Arntzen KA, Schirmer $\mathrm{H}$, Wilsgaard T, Mathiesen EB. Impact of cardiovascular risk factors on cognitive function: the Tromso study. Eur J Neurol. 2011;18(5):737-43.

6. Yaffe K, Bahorik AL, Hoang TD, Forrester S, Jacobs DR Jr, Lewis CE, et al. Cardiovascular risk factors and accelerated cognitive decline in midlife: The CARDIA Study. Neurology. 2020;95(7):e839-e46.

7. Schreiner PJ, Jacobs DR Jr, Wong ND, Kiefe Cl. Twenty-five year secular trends in lipids and modifiable risk Factors in a population-based biracial cohort: The Coronary Artery Risk Development in Young Adults (CARDIA) Study, 1985-2011. Journal of the American Heart Association. 2016;5(7).

8. Unverzagt FW, McClure LA, Wadley VG, Jenny NS, Go RC, Cushman M, et al. Vascular risk factors and cognitive impairment in a stroke-free cohort. Neurology. 2011;77(19):1729-36.

9. Howard VJ, Cushman M, Pulley L, Gomez CR, Go RC, Prineas RJ, et al. The reasons for geographic and racial differences in stroke study: objectives and design. Neuroepidemiology. 2005;25(3):13543.

10. van der Lee SJ, Teunissen CE, Pool R, Shipley MJ, Teumer A, Chouraki V, et al. Circulating metabolites and general cognitive ability and dementia: Evidence from 11 cohort studies. Alzheimers Dement. 2018;14(6):707-22.

11. Frank AT, Zhao B, Jose PO, Azar KM, Fortmann SP, Palaniappan LP. Racial/ethnic differences in dyslipidemia patterns. Circulation. 2014;129(5):570-9.

12. Liu L, Zhang C, Lv X, Lai X, Xu L, Feng J, et al. Sex-specific associations between lipids and cognitive decline in the middle-aged and elderly: a cohort study of Chinese adults. Alzheimer's research therapy. 2020;12(1):164.

13. Nordestgaard LT, Christoffersen M, Afzal S, Nordestgaard BG, Tybjærg-Hansen A, Frikke-Schmidt R. Triglycerides as a shared risk factor between dementia and atherosclerotic cardiovascular disease: a study of 125727 individuals. Clinical chemistry. 2021;67(1):245-55.

14. Rosenson RS, Brewer HB Jr, Barter PJ, Björkegren JLM, Chapman MJ, Gaudet D, et al. HDL and atherosclerotic cardiovascular disease: genetic insights into complex biology. Nature reviews Cardiology. 2018;15(1):9-19.

15. Bahrami A, Barreto GE, Lombardi G, Pirro M, Sahebkar A. Emerging roles for high-density lipoproteins in neurodegenerative disorders. Biofactors. 2019;45(5):725-39. 